\title{
Multiplicity result for a critical elliptic system with concave-convex nonlinearities
}

\section{Cyril Joël Batkam ${ }^{1}$ and Fabrice Colin²}

${ }^{*}$ Correspondence:

fcolin@cs.laurentian.ca

${ }^{2}$ Mathematics and Computer

Science Department, Laurentian

University, Sudbury, Ontario P3E 2C6, Canada

Full list of author information is available at the end of the article

\section{Abstract}

We study the existence of multiple solutions of a strongly indefinite elliptic system involving the critical Sobolev exponent and concave-convex nonlinearities. By using a suitable version of the dual fountain theorem established in this paper, we prove the existence of infinitely many small energy solutions.

MSC: $35 \mathrm{~A} 02 ; 35 J 50 ; 35 J 60$

Keywords: critical nonlinearity; concave-convex nonlinearity; noncooperative elliptic system; dual fountain theorem; small energy solutions

\section{Introduction}

In this paper, we study the existence and multiplicity of solutions for the following elliptic system of noncooperative type:

$$
\left\{\begin{array}{l}
-\Delta u=\lambda|u|^{p-2} u-\frac{\alpha}{\alpha+\beta}|u|^{\alpha-2} u|v|^{\beta} \quad \text { in } \Omega \\
\Delta v=\mu|v|^{q-2} v-\frac{\beta}{\alpha+\beta}|u|^{\alpha}|v|^{\beta-2} v \quad \text { in } \Omega \\
\left.u\right|_{\partial \Omega}=\left.v\right|_{\partial \Omega}=0
\end{array}\right.
$$

where $\Omega$ is a bounded smooth domain in $\mathbb{R}^{N}$ with $N \geq 3, \lambda>0$, and $\mu>0,1<p, q<2$ and $\alpha, \beta>1$ satisfy $\alpha+\beta=2^{\star}$, where $2^{\star}:=2 N / N-2$ denotes the critical Sobolev exponent. The solutions of $\left(S_{\lambda, \mu}\right)$ are steady states of reaction-diffusion systems, which serve as a class of models with applications in physics, chemistry and biology (see, for instance, $[1,2]$ ).

It is well known that weak solutions of $\left(S_{\lambda, \mu}\right)$ are critical points of the following functional defined on the Hilbert space $H_{0}^{1}(\Omega) \times H_{0}^{1}(\Omega)$ :

$$
J(u, v):=\int_{\Omega}\left[\frac{1}{2}\left(|\nabla u|^{2}-|\nabla v|^{2}\right)-\frac{\lambda}{p}|u|^{p}-\frac{\mu}{q}|v|^{q}+\frac{1}{\alpha+\beta}|u|^{\alpha}|v|^{\beta}\right] \mathrm{d} x .
$$

We call $(u, v)$ a weak solution of $\left(S_{\lambda, \mu}\right)$ if $(u, v) \in H_{0}^{1}(\Omega) \times H_{0}^{1}(\Omega)$ and

$$
\begin{aligned}
& \int_{\Omega}\left[\nabla u \nabla \phi-\nabla v \nabla \varphi-\lambda|u|^{p-2} u \phi-\mu|v|^{q-2} \nu \varphi\right. \\
& \left.\quad+\frac{1}{\alpha+\beta}\left(\alpha|u|^{\alpha-2}|\nu|^{\beta} u \phi+\beta|u|^{\alpha}|\nu|^{\beta-2} \nu \varphi\right)\right] \mathrm{d} x=0
\end{aligned}
$$

for every $(\phi, \varphi) \in H_{0}^{1}(\Omega) \times H_{0}^{1}(\Omega)$.

\section{Springer}

○2013 Batkam and Colin; licensee Springer. This is an Open Access article distributed under the terms of the Creative Commons Attribution License (http://creativecommons.org/licenses/by/2.0), which permits unrestricted use, distribution, and reproduction in any medium, provided the original work is properly cited. 
The celebrated papers by Brezis and Nirenberg [3] and by Ambrosetti et al. [4] have inspired research on differential equations and systems with critical terms, and with concave-convex nonlinearities, respectively, see, for example, [5-12] and the many references therein. In recent years, more and more attention have been paid to the existence and multiplicity of solutions of the following elliptic system of cooperative type:

$$
\left\{\begin{array}{l}
-\Delta u=\lambda|u|^{q-2} u+\frac{2 \alpha}{\alpha+\beta}|u|^{\alpha-2} u|v|^{\beta} \quad \text { in } \Omega \\
-\Delta v=\mu|v|^{q-2} v+\frac{2 \beta}{\alpha+\beta}|u|^{\alpha}|v|^{\beta-2} v \quad \text { in } \Omega \\
\left.u\right|_{\partial \Omega}=\left.v\right|_{\partial \Omega}=0 .
\end{array}\right.
$$

In [9], by extracting Palais-Smale sequences on the Nehari manifold, Hsu and Lin proved that if $\lambda$ and $\mu$ are small, then $\left(P_{\lambda, \mu}\right)$ has at least two solutions. Subsequently, their result was extended by Chen and $\mathrm{Wu}$ [13], who considered $\left(P_{\lambda, \mu}\right)$ with sign-changing weight functions. See also $[12,14]$ and the references therein for some related results.

However, what can be said about the noncooperative case? To the best of our knowledge, there is no result on problem $\left(S_{\lambda, \mu}\right)$, even in the subcritical case $\alpha+\beta<2^{\star}$. In addition to the combined effects of the concave and convex terms, two main difficulties arise when studying $\left(S_{\lambda, \mu}\right)$. The first one is that the energy functional $J$ above is strongly indefinite in the sense that it is neither bounded from above nor from below, even on subspaces of finite codimension. Therefore, the usual critical point theorems such as the mountain pass theorem cannot be used. Moreover, the method of the Nehari manifold, which is extensively used in the cooperative case does not apply in this situation. We refer to [15] for a unified approach on the method of the Nehari manifold. The second difficulty arises with the critical Sobolev exponent. Indeed, it is well known that the embedding $H_{0}^{1}(\Omega) \hookrightarrow$ $L^{2^{\star}}(\Omega)$ is not compact because of the action of dilatations. Therefore, the energy functional is not expected to satisfy the Palais-Smale condition (see Definition 5 below ). Usually, the best we can expect is to find a bounded from above subset $I \subset \mathbb{R}$ such that the functional satisfies the Palais-Smale condition at every level $c \in I$, see, for instance, $[3,9,16,17]$. But surprisingly enough, we will show that in our case the functional $J$ satisfies the PalaisSmale condition.

The main result of the paper is the following.

Theorem 1 Let $\lambda, \mu>0$. Then problem $\left(S_{\lambda, \mu}\right)$ has a sequence of solutions $\left(u_{k}, v_{k}\right)$ such that $J\left(u_{k}, v_{k}\right)<0$ and $J\left(u_{k}, v_{k}\right) \rightarrow 0$ as $k \rightarrow \infty$.

Remark 2 Theorem 1 remains true in the subcritical case, i.e., if we assume that $\alpha+\beta>2$ if $N=1,2$ or $2<\alpha+\beta<2^{\star}$ if $N=3$. In this case, the proof of Theorem 1 is considerably simplified, since the embedding $H_{0}^{1}(\Omega) \hookrightarrow L^{\alpha+\beta}(\Omega)$ is now compact.

The paper is organized as follows. In order to prove Theorem 1, we need a new critical point theorem for strongly indefinite functionals, which will be provided in Section 2. This critical point theorem generalizes the dual fountain theorem of Bartsch and Willem, and is not based on any reduction method. It should be noted that problem $\left(S_{\lambda, \mu}\right)$ does not fit into the framework of the theorem proved in [18], which is only suitable for finding large energy solutions. Finally the proof of our main result is presented in Section 3. 


\section{Critical point theory}

Let $Y$ be a closed subspace of a separable Hilbert space $X$ endowed with the inner product $(\cdot)$ and the associated norm $\|\cdot\|$. We denote by $P: X \rightarrow Y$ and $Q: X \rightarrow Z:=Y^{\perp}$ the orthogonal projections.

We fix an orthonormal basis $\left(e_{j}\right)_{j \geq 0}$ of $Y$, and we consider on $X=Y \oplus Z$ the $\tau$-topology introduced by Kryszewski and Szulkin in [19]; that is, the topology associated to the following norm

$$
\|u\| \|:=\max \left(\sum_{j=0}^{\infty} \frac{1}{2^{j+1}}\left|\left(P u, e_{j}\right)\right|,\|Q u\|\right), \quad u \in X .
$$

Clearly $\|Q u\| \leq\|u\| \leq\|u\|$. Moreover, if $\left(u_{n}\right)$ is a bounded sequence in $X$, then

$$
u_{n} \stackrel{\tau}{\rightarrow} u \Longleftrightarrow P u_{n} \rightarrow P u \quad \text { and } \quad Q u_{n} \rightarrow Q u
$$

Definition 3 Let $\Phi \in \mathcal{C}^{1}(X, \mathbb{R})$ and $a, b \in \mathbb{R}, a<b$. We say that $\Phi^{\prime}$ is $\tau$-weak sequentially compact in $\Phi^{-1}([a, b])$ if for every sequence $u_{n}$ which $\tau$-converges to $u$ in $\Phi^{-1}([a, b])$, there is a subsequence $\left(u_{n_{k}}\right)$ such that $\Phi^{\prime}\left(u_{n_{k}}\right) \rightarrow \Phi^{\prime}(u)$.

Lemma 4 (Deformation lemma) Let $\varphi \in \mathcal{C}^{1}(X, \mathbb{R})$ be an even functional which is $\tau$-upper semicontinuous, and such that $\nabla \varphi$ is $\tau$-weak sequentially compact in $\varphi^{-1}([a, b])$ for any $a, b \in \mathbb{R}$. Let $S \subset X$ with $-S=S$, and $c \in \mathbb{R}, \varepsilon, \theta>0$ such that

$$
\forall u \in \varphi^{-1}([c-2 \varepsilon, c+2 \varepsilon]) \cap S_{2 \theta}, \quad\left\|\varphi^{\prime}(u)\right\| \geq \frac{8 \varepsilon}{\theta} .
$$

Then there exists $\eta \in \mathcal{C}\left([0,1] \times \varphi^{c+2 \varepsilon}, X\right)$ such that

(i) $\eta(t, u)=u$ if $t=0$ or if $u \notin \varphi^{-1}([c-2 \varepsilon, c+2 \varepsilon]) \cap S_{2 \theta}$,

(ii) $\eta\left(1, \varphi^{c+\varepsilon} \cap S\right) \subset \varphi^{c-\varepsilon}$,

(iii) $\|\eta(t, u)-u\| \leq \frac{\theta}{2} \forall u \in \varphi^{c+2 \varepsilon}, \forall t \in[0,1]$,

(iv) $\varphi(\eta(\cdot, u))$ is non increasing, $\forall u \in \varphi^{c+2 \varepsilon}$,

(v) Each point $(t, u) \in[0,1] \times \varphi^{c+2 \varepsilon}$ has a $\tau$-neighborhood $N_{(t, u)}$ such that

$\left\{v-\eta(s, v) \mid(s, v) \in N_{(t, u)} \cap\left([0,1] \times \varphi^{c+2 \varepsilon}\right)\right\}$ is contained in a finite-dimensional subspace of $X$,

(vi) $\eta$ is $\tau$-continuous,

(vii) $\eta(t, \cdot)$ is odd $\forall t \in[0,1]$,

where $S_{\alpha}:=\{u \in X \mid \operatorname{dist}(u, S) \leq \alpha\} \forall \alpha>0$ and $\varphi^{a}:=\{u \in X \mid \varphi(u) \leq a\} \forall a \in \mathbb{R}$.

Proof We define

$$
w(v):=2\|\nabla \varphi(v)\|^{-2} \nabla \varphi(v), \quad \forall v \in \varphi^{-1}([c-2 \varepsilon, c+2 \varepsilon]) .
$$

We claim that for every $v \in \varphi^{-1}([c-2 \varepsilon, c+2 \varepsilon])$, there exists a $\tau$-open neighborhood $N_{\nu}$ of $v$ such that $(\nabla \varphi(u), w(v))>1 \forall u \in N_{v}\left(N_{\nu}\right.$ can be chosen to be symmetric, that is, $\left.-N_{v}=N_{v}\right)$. In fact, if this is not true, then we can find a sequence $\left(v_{n}\right) \subset \varphi^{-1}([c-2 \varepsilon, c+2 \varepsilon])$ which $\tau$ converges to an element $v \in \varphi^{-1}([c-2 \varepsilon, c+2 \varepsilon])$ and such that $\left(\nabla \varphi\left(v_{n}\right), w(v)\right) \leq 1$. Since $\nabla \varphi$ is $\tau$-weak sequentially compact, there is a subsequence $\left(v_{n_{k}}\right)$ such that $\nabla \varphi\left(v_{n_{k}}\right) \rightarrow \nabla \varphi(v)$, and this implies that $(\nabla \varphi(v), w(v)) \leq 1$, which is in contradiction with the definition of $w$. 
The rest of the proof follows the same lines as the proof of Lemma 8 in [18] with $G=\mathbb{Z}_{2}$.

We introduce the following notations:

$$
\begin{aligned}
& Y_{k}:=\bigoplus_{j=k}^{\infty} \mathbb{R} e_{j}, \quad Z_{k}:=\left(\bigoplus_{j=0}^{k} \mathbb{R} e_{j}\right) \oplus Z, \\
& B_{k}:=\left\{u \in Y_{k} \mid\|u\| \leq \sigma_{k}\right\}, \quad N_{k}:=\left\{u \in Z_{k} \mid\|u\|=s_{k}\right\}, \quad \text { with } 0<s_{k}<\sigma_{k}, k \geq 2 .
\end{aligned}
$$

Before we state and prove our critical point theorem, we recall the following definition.

Definition 5 A functional $\Phi \in \mathcal{C}^{1}(X, \mathbb{R})$ is said to satisfy the Palais-Smale condition (resp. $(P S)_{c}$ condition ) if every sequence $\left(u_{n}\right) \subset X$ such that $\left(\Phi\left(u_{n}\right)\right)$ is bounded (resp. $\Phi\left(u_{n}\right) \rightarrow c$ ) and $\Phi^{\prime}\left(u_{n}\right) \rightarrow 0$, has a convergent subsequence.

Theorem 6 (Generalized dual fountain theorem) Let $\Phi \in \mathcal{C}^{1}(X, \mathbb{R})$ be an even functional which is $\tau$-lower semicontinuous and such that $\nabla \Phi$ is $\tau$-weak sequentially compact in $\Phi^{-1}([a, b])$ for any $a, b \in \mathbb{R}$. If, for every $k \geq k_{0}$, there exist $\sigma_{k}>s_{k}>0$ such that

$\left(\mathrm{B}_{1}\right) \quad a_{k}:=\inf _{\| \in Y_{k}} \Phi(u) \geq 0$,

$\left(\mathrm{B}_{2}\right) \quad b_{k}:=\sup _{\|u\|=Z_{k}} \Phi(u)<0$,

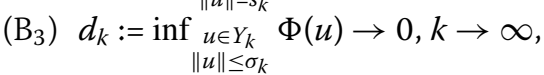

$\left(\mathrm{B}_{4}\right) \Phi$ satisfies the $(P S)_{c}$ condition $\forall c \in\left[d_{k_{0}}, 0[\right.$.

Then $\Phi$ has a sequence of critical points $\left(u_{k}\right)$ such that $\Phi\left(u_{k}\right)<0$ and $\Phi\left(u_{k}\right) \rightarrow 0$ as $k \rightarrow \infty$.

Proof Let $\Gamma_{k}$ be the set of maps $\gamma: B_{k} \rightarrow X$ such that

(a) $\gamma$ is odd, $\tau$-continuous and $\left.\gamma\right|_{\partial B_{k}}=\mathrm{id}$,

(b) each $u \in \operatorname{int}\left(B_{k}\right)$ has a $\tau$-neighborhood $\mathcal{N}_{u}$ in $Y_{k}$ such that $(\operatorname{id}-\gamma)\left(\mathcal{N}_{u} \cap \operatorname{int}\left(B_{k}\right)\right)$ is contained in a finite dimensional subspace of $X$,

(c) $\Phi(\gamma(u)) \geq \Phi(u) \forall u \in B_{k}$.

Define

$$
c_{k}:=\sup _{\gamma \in \Gamma_{k}} \inf _{u \in B_{k}} \Phi(\gamma(u))
$$

It follows from the definitions that $d_{k} \leq c_{k}$. Lemma 10 in [18] implies that $\gamma\left(B_{k}\right) \cap N_{k} \neq \emptyset$ $\forall \gamma \in \Gamma_{k}$, which in turn implies that $c_{k} \leq b_{k}$.

Let $\varepsilon \in] 0, \frac{a_{k}-c_{k}}{2}\left[, \theta>0\right.$, and let $\gamma \in \Gamma_{k}$ such that

$$
c_{k}-\varepsilon \leq \inf _{u \in B_{k}} \Phi(\gamma(u))
$$

We claim that

$$
\exists u \in \Phi^{-1}\left(\left[c_{k}-2 \varepsilon, c_{k}+2 \varepsilon\right]\right) \cap\left(\gamma\left(B_{k}\right)\right)_{2 \theta} \quad \text { such that }\left\|\Phi^{\prime}(u)\right\| \leq \frac{8 \varepsilon}{\theta} .
$$


Hence, there exists a sequence $\left(u_{n}\right) \subset X$ such that

$$
\Phi\left(u_{n}\right) \rightarrow c_{k} \quad \text { and } \quad \Phi^{\prime}\left(u_{n}\right) \rightarrow 0, \quad n \rightarrow \infty .
$$

The conclusion of Theorem 6 then follows from $\left(B_{4}\right)$ and $\left(B_{3}\right)$.

To complete the proof of Theorem 6, it remains to show that (3) holds.

We proceed by contradiction by assuming that (3) does not hold, and then, we apply Lemma 4 with $\varphi=-\Phi, c=-c_{k}$ and $S=\gamma\left(B_{k}\right)$. We may assume that $c_{k}+2 \varepsilon<a_{k}$. Next, by following the proof of Theorem 11 in [18], one can easily verify that the map $\beta$ defined on $B_{k}$ by $\beta(u):=\eta(1, \gamma(u))$ belongs to $\Gamma_{k}$. Now, (2) and (ii) of Lemma 4 imply that

$$
\inf _{u \in B_{k}} \Phi(\beta(u)) \geq c_{k}+\varepsilon
$$

which contradicts the definition of $c_{k}$.

\section{Proof of the main results}

In this section, we denote by $|u|_{p}$ the usual $L^{p}(\Omega)$ norm.

We consider the Sobolev space $H_{0}^{1}(\Omega)$ endowed with the norm $\|u\|=|\nabla u|_{2}$ and $X:=$ $H_{0}^{1}(\Omega) \times H_{0}^{1}(\Omega)$ with the product norm $\|(u, v)\|=\left(\|u\|^{2}+\|v\|^{2}\right)^{\frac{1}{2}}$.

The functional $J$ defined by (1) then reads as follows:

$$
J(u, v):=\frac{1}{2}\|u\|^{2}-\frac{1}{2}\|v\|^{2}-\frac{\lambda}{p}|u|_{p}^{p}-\frac{\mu}{q}|v|_{q}^{q}+\frac{1}{\alpha+\beta} \int_{\Omega}|u|^{\alpha}|v|^{\beta} \mathrm{d} x .
$$

A standard argument shows that $J$ is of $\operatorname{class} \mathcal{C}^{1}$ on $X$ and

$$
\begin{aligned}
\left\langle J^{\prime}(u, v),(\phi, \varphi)\right\rangle= & \int_{\Omega}\left[\nabla u \nabla \phi-\nabla \nu \nabla \varphi-\lambda|u|^{p-2} u \phi-\mu|\nu|^{q-2} \nu \varphi\right. \\
& \left.+\frac{1}{\alpha+\beta}\left(\alpha|u|^{\alpha-2}|\nu|^{\beta} u \phi+\beta|u|^{\alpha}|\nu|^{\beta-2} v \varphi\right)\right] \mathrm{d} x .
\end{aligned}
$$

Lemma 7 Let $\left(u_{n}, v_{n}\right) \subset X$ such that $\left(u_{n}, v_{n}\right) \rightarrow(u, v)$ in $X$. Then there exists a subsequence $\left(u_{n_{k}}, v_{n_{k}}\right)$ such that $J^{\prime}\left(u_{n_{k}}, v_{n_{k}}\right) \rightarrow J^{\prime}(u, v)$.

Proof Let $(\phi, \varphi) \in X$. Using (5), we have

$$
\begin{aligned}
\left\langle J^{\prime}\left(u_{n}, v_{n}\right)-J^{\prime}(u, v),(\phi, \varphi)\right\rangle= & \int_{\Omega}\left[\nabla\left(u_{n}-u\right) \nabla \phi-\nabla\left(v_{n}-v\right) \nabla \varphi\right. \\
& -\lambda\left(\left|u_{n}\right|^{p-2} u_{n}-|u|^{p-2} u\right) \phi-\mu\left(\left|v_{n}\right|^{q-2} v_{n}-|v|^{q-2} v\right) \varphi \\
& +\frac{\alpha}{\alpha+\beta}\left(\left|u_{n}\right|^{\alpha-2} u_{n}\left|v_{n}\right|^{\beta}-|u|^{\alpha-2} u|v|^{\beta}\right) \phi \\
& \left.+\frac{\beta}{\alpha+\beta}\left(\left|u_{n}\right|^{\alpha}\left|v_{n}\right|^{\beta-2} v_{n}-|u|^{\alpha}|v|^{\beta-2} v\right) \varphi\right] \mathrm{d} x .
\end{aligned}
$$

It is clear that

$$
\int_{\Omega} \nabla\left(u_{n}-u\right) \nabla \phi \mathrm{d} x \rightarrow 0 \quad \text { and } \quad \int_{\Omega} \nabla\left(v_{n}-v\right) \nabla \varphi \mathrm{d} x \rightarrow 0 .
$$


Now up to a subsequence we have

$$
u_{n} \rightarrow u \text { and } \quad v_{n} \rightarrow v \text { a.e. on } \Omega \text {. }
$$

By the Sobolev embedding theorem $\left(u_{n}\right)$ and $\left(v_{n}\right)$ are bounded in $L^{p}(\Omega), L^{q}(\Omega)$ and $L^{\alpha+\beta}(\Omega)$. So $\left(\left|u_{n}\right|^{p-2} u_{n}\right)$ (resp. $\left(\left|v_{n}\right|^{q-2} v_{n}\right)$ ) is bounded in $L^{\frac{p}{p-1}}(\Omega)$ (resp. in $L^{\frac{q}{q-1}}(\Omega)$ ), $\left(\left|u_{n}\right|^{\alpha-2} u_{n}\left|v_{n}\right|^{\beta}\right)$ and $\left(\left|u_{n}\right|^{\alpha}\left|v_{n}\right|^{\beta-2} v_{n}\right)$ are bounded in $L^{\frac{\alpha+\beta}{\alpha+\beta-1}}(\Omega)$.

By Theorem 10.36 in [20], we have

$$
\begin{aligned}
& \left|u_{n}\right|^{p-2} u_{n} \rightarrow|u|^{p-2} u \quad \text { in } L^{\frac{p}{p-1}}(\Omega), \quad\left|v_{n}\right|^{q-2} v_{n} \rightarrow|v|^{q-2} v \quad \text { in } L^{\frac{q}{q-1}}(\Omega), \\
& \left|u_{n}\right|^{\alpha-2} u_{n}\left|v_{n}\right|^{\beta} \rightarrow|u|^{\alpha-2} u|v|^{\beta} \quad \text { and } \quad\left|u_{n}\right|^{\alpha}\left|v_{n}\right|^{\beta-2} v_{n} \rightarrow|u|^{\alpha}|v|^{\beta-2} v \quad \text { in } L^{\frac{\alpha+\beta}{\alpha+\beta-1}}(\Omega) .
\end{aligned}
$$

Since $\phi, \varphi \in H_{0}^{1}(\Omega) \subset L^{p}(\Omega) \cap L^{q}(\Omega) \cap L^{\alpha+\beta}(\Omega)$, it follows that

$$
\begin{aligned}
& \int_{\Omega}\left(\left|u_{n}\right|^{p-2} u_{n}-|u|^{p-2} u\right) \phi \mathrm{d} x \rightarrow 0, \quad \int_{\Omega}\left(\left|v_{n}\right|^{q-2} v_{n}-|v|^{q-2} v\right) \varphi \mathrm{d} x \rightarrow 0, \\
& \int_{\Omega}\left(\left|u_{n}\right|^{\alpha-2} u_{n}\left|v_{n}\right|^{\beta}-|u|^{\alpha-2} u|v|^{\beta}\right) \phi \mathrm{d} x \rightarrow 0, \\
& \int_{\Omega}\left(\left|u_{n}\right|^{\alpha}\left|v_{n}\right|^{\beta-2} v_{n}-|u|^{\alpha}|v|^{\beta-2} v\right) \varphi \mathrm{d} x \rightarrow 0,
\end{aligned}
$$

and $\left\langle J^{\prime}\left(u_{n}, v_{n}\right)-J^{\prime}(u, v),(\phi, \varphi)\right\rangle \rightarrow 0$ for every $(\phi, \varphi) \in X$. Hence, $J^{\prime}\left(u_{n}, v_{n}\right) \rightarrow J^{\prime}(u, v)$.

Lemma 8 The functional J satisfies the Palais-Smale condition.

Proof Let $\left(u_{n}, v_{n}\right) \subset X$ such that

$$
d:=\sup _{n}\left|J\left(u_{n}, v_{n}\right)\right|<\infty \quad \text { and } \quad J^{\prime}\left(u_{n}, v_{n}\right) \rightarrow 0, \quad n \rightarrow \infty .
$$

We deduce from (5) that

$$
\begin{aligned}
\left\langle J^{\prime}\left(u_{n}, v_{n}\right),\left(u_{n}, 0\right)\right\rangle & =\left\|u_{n}\right\|^{2}-\lambda\left|u_{n}\right|_{p}^{p}+\frac{\alpha}{\alpha+\beta} \int_{\Omega}\left|u_{n}\right|^{\alpha}\left|v_{n}\right|^{\beta} \mathrm{d} x \\
& \geq\left\|u_{n}\right\|^{2}-\lambda\left|u_{n}\right|_{p}^{p} .
\end{aligned}
$$

Hence for $n$ big enough, we have

$$
\left\|u_{n}\right\|^{2}-\lambda\left|u_{n}\right|_{p}^{p} \leq\left\|u_{n}\right\|
$$

which implies, since $p<2$, that $\left(u_{n}\right)$ is bounded.

On the other hand, by using (4) and (5), we obtain

$$
\begin{aligned}
-J\left(u_{n}, v_{n}\right)+\frac{1}{\alpha+\beta}\left\langle J^{\prime}\left(u_{n}, v_{n}\right),\left(u_{n}, v_{n}\right)\right\rangle= & \left(\frac{1}{\alpha+\beta}-\frac{1}{2}\right)\left\|u_{n}\right\|^{2}+\left(\frac{1}{2}-\frac{1}{\alpha+\beta}\right)\left\|v_{n}\right\|^{2} \\
& +\lambda\left(\frac{1}{p}-\frac{1}{\alpha+\beta}\right)\left|u_{n}\right|_{p}^{p}+\mu\left(\frac{1}{q}-\frac{1}{\alpha+\beta}\right)\left|v_{n}\right|_{q}^{q} .
\end{aligned}
$$


Since $\left(u_{n}\right)$ is bounded, $q<2<\alpha+\beta$ and

$$
-J\left(u_{n}, v_{n}\right)+\frac{1}{\alpha+\beta}\left\langle J^{\prime}\left(u_{n}, v_{n}\right),\left(u_{n}, v_{n}\right)\right\rangle \leq d+\left\|\left(u_{n}, v_{n}\right)\right\|
$$

for $n$ big enough, we easily deduce that $\left(v_{n}\right)$ is bounded.

Consequently, we have, up to a subsequence,

$$
\begin{aligned}
& \left(u_{n}, v_{n}\right) \rightarrow(u, v) \quad \text { in } X, \\
& u_{n} \rightarrow u \quad \text { in } L^{p}(\Omega), \quad v_{n} \rightarrow v \quad \text { in } L^{q}(\Omega), \\
& u_{n} \rightarrow u, \quad v_{n} \rightarrow v \quad \text { a.e. on } \Omega, \\
& \left.\nabla J\left(u_{n}, v_{n}\right) \rightarrow \nabla J(u, v) \quad \text { (by Lemma } 7\right) .
\end{aligned}
$$

On the other hand, $J^{\prime}\left(u_{n}, v_{n}\right) \rightarrow 0$ and equation (6) imply that $J^{\prime}(u, v)=0$ and that

$$
\left\langle J^{\prime}\left(u_{n}, v_{n}\right),\left(u_{n}, 0\right)\right\rangle-\left\langle J^{\prime}(u, v),(u, 0)\right\rangle \rightarrow 0, \quad\left\langle J^{\prime}\left(u_{n}, v_{n}\right),\left(0, v_{n}\right)\right\rangle-\left\langle J^{\prime}(u, v),(0, v)\right\rangle \rightarrow 0
$$

Now, because of equation (7), it follows that

$$
\begin{aligned}
& \left\|u_{n}\right\|^{2}-\|u\|^{2}+\frac{\alpha}{\alpha+\beta} \int_{\Omega}\left(\left|u_{n}\right|^{\alpha}\left|v_{n}\right|^{\beta}-|u|^{\alpha}|v|^{\beta}\right) \mathrm{d} x \rightarrow 0, \\
& -\left\|v_{n}\right\|^{2}+\|v\|^{2}+\frac{\beta}{\alpha+\beta} \int_{\Omega}\left(\left|u_{n}\right|^{\alpha}\left|v_{n}\right|^{\beta}-|u|^{\alpha}|v|^{\beta}\right) \mathrm{d} x \rightarrow 0 .
\end{aligned}
$$

Let us combine the preceding equations in order to cancel the integrals. We then get

$$
\beta\left(\left\|u_{n}\right\|^{2}-\|u\|^{2}\right)+\alpha\left(\left\|v_{n}\right\|^{2}-\|v\|^{2}\right) \rightarrow 0
$$

But since equation (6) leads to

$$
\left\|u_{n}\right\|^{2}=\left\|u_{n}-u\right\|^{2}+\|u\|^{2}+\circ(1), \quad\left\|v_{n}\right\|^{2}=\left\|v_{n}-v\right\|^{2}+\|v\|^{2}+\circ(1)
$$

it is then easy to conclude that

$$
\beta\left\|u_{n}-u\right\|^{2}+\alpha\left\|v_{n}-v\right\|^{2} \rightarrow 0
$$

Hence $\left(u_{n}, v_{n}\right) \rightarrow(u, v)$.

Now, we select an orthonormal basis $\left(e_{j}\right)$ of $H_{0}^{1}(\Omega)$, and we consider the $\tau$-topology on $X=Y \oplus Z$, where $Y$ and $Z$ are defined by

$$
Y:=H_{0}^{1}(\Omega) \times\{0\} \quad \text { and } \quad Z:=\{0\} \times H_{0}^{1}(\Omega) \text {. }
$$

Lemma $9 J$ is $\tau$-lower semicontinuous, and $\nabla J$ is $\tau$-weak sequentially compact in $J^{-1}([a, b])$ for any $a, b \in \mathbb{R}$. 


\section{Proof}

1. Let $\left(u_{n}, v_{n}\right) \subset X$ and $C \in \mathbb{R}$ such that $\left(u_{n}, v_{n}\right) \stackrel{\tau}{\rightarrow}(u, v)$ in $X$ and $J\left(u_{n}, v_{n}\right) \leq C$. By the definition of $\tau,\left(v_{n}\right)$ converges strongly to $v$ in $H_{0}^{1}(\Omega)$. Clearly, we have

$$
J\left(u_{n}, v_{n}\right) \geq \frac{1}{2}\left\|u_{n}\right\|^{2}-\frac{1}{2}\left\|v_{n}\right\|^{2}-\frac{\lambda}{p}\left|u_{n}\right|_{p}^{p}-\frac{\mu}{q}\left|v_{n}\right|_{q}^{q},
$$

which implies, since $\left(v_{n}\right)$ is bounded and $p<2$, that $\left(u_{n}\right)$ is bounded. Up to a subsequence, we may assume that $u_{n} \rightarrow u$ in $L^{p}(\Omega)$ and $v_{n} \rightarrow v$ in $L^{q}(\Omega)$,

$u_{n}(x) \rightarrow u(x)$ and $v_{n}(x) \rightarrow v(x)$ for almost every $x \in \Omega$. By using Fatou's lemma and the weak lower semicontinuity of the norm $\|\cdot\|$, we deduce from the inequality

$$
C \geq J\left(u_{n}, v_{n}\right)=\frac{1}{2}\left\|u_{n}\right\|^{2}-\frac{1}{2}\left\|v_{n}\right\|^{2}-\frac{\lambda}{p}\left|u_{n}\right|_{p}^{p}-\frac{\mu}{q}\left|v_{n}\right|_{q}^{q}+\frac{1}{\alpha+\beta} \int_{\Omega}\left|u_{n}\right|^{\alpha}\left|v_{n}\right|^{\beta} \mathrm{d} x
$$

that $C \geq J(u, v)$.

2. Assume that $\left(u_{n}, v_{n}\right) \stackrel{\tau}{\rightarrow}(u, v)$ in $J^{-1}([a, b]), a, b \in \mathbb{R}$. The same argument as above shows that $\left(u_{n}, v_{n}\right)$ is bounded, and then $\left(u_{n}, v_{n}\right) \rightarrow(u, v)$. It follows from Lemma 7 that $\nabla J$ is $\tau$-weak sequentially compact in $J^{-1}([a, b])$.

Proof of Theorem 1 We recall that

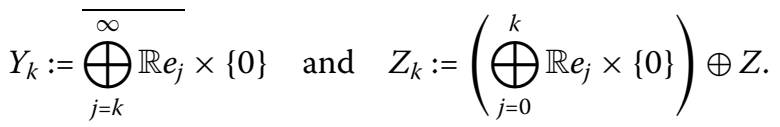

Let $(u, 0) \in Y_{k}$. Then, since $\lambda>0$, we have

$$
J(u, 0)=\frac{1}{2}\|u\|^{2}-\frac{\lambda}{p}|u|_{p}^{p} \geq \frac{1}{2}\|u\|^{2}-\frac{\lambda}{p} \theta_{k}^{p}\|u\|^{p},
$$

where

$$
\theta_{k}=\sup _{\substack{w \in \bigoplus_{j=k}^{\infty} \mathbb{R e} e_{j} \\\|w\|=1}}|w|_{p}
$$

Therefore, for every $(u, 0) \in Y_{k}$ such that $\|u\|=\sigma_{k}:=2\left(\frac{2 \lambda}{p} \theta_{k}^{p}\right)^{\frac{1}{2-p}}$, we have

$$
J(u, 0) \geq\left(\frac{\lambda}{p} \theta_{k}^{p}\right)^{\frac{2}{2-p}} 2^{\frac{p}{2-p}}\left(2^{2}-2^{p}\right)>0 .
$$

On the other hand, it is clear that

$$
\frac{1}{2}\|u\|^{2} \geq J(u, 0) \geq-\frac{\lambda}{p} \theta_{k}^{p}\|u\|^{p} .
$$

Hence, for every $(u, 0) \in Y_{k}$ such that $\|u\| \leq \sigma_{k}$, we have

$$
\frac{1}{2} \sigma_{k}^{2} \geq J(u, 0) \geq-\frac{\lambda}{p} \theta_{k}^{p} \sigma_{k}^{p} .
$$

We know by [21] that $\theta_{k} \rightarrow 0$ as $k \rightarrow \infty$, hence we deduce that $J(u, 0) \rightarrow 0$ as $k \rightarrow \infty$. 
We have then proved that assumptions $\left(\mathrm{B}_{1}\right)$ and $\left(\mathrm{B}_{3}\right)$ of Theorem 6 are satisfied.

Let $(u, v) \in Z_{k}$. Since the norms $\|\cdot\|$ and $|\cdot|_{p}$ are equivalent on $\bigoplus_{j=0}^{k} \mathbb{R} e_{j}, \mu>0$ and $H_{0}^{1}(\Omega)$ continuously embeds into $L^{\alpha+\beta}(\Omega)$, we have

$$
J(u, v) \leq \frac{1}{2}\|u\|^{2}-\frac{\lambda c_{1}}{p}\|u\|^{p}+\frac{c}{\alpha+\beta}\|u\|^{\alpha+\beta}-\frac{1}{2}\|v\|^{2}+\frac{c}{\alpha+\beta}\|v\|^{\alpha+\beta},
$$

where $c_{1}>0$ and $c>0$ are constants. It is then easy to verify, since $\lambda>0$, that assumption $\left(\mathrm{B}_{2}\right)$ of Theorem 6 is satisfied for $s_{k}$ small enough.

By using Lemmas 8, 9, we can apply Theorem 6 and get the desired result.

\section{Competing interests}

The authors declare that they have no competing interests.

\section{Authors' contributions}

All authors contributed equally to the manuscript. In addition, both authors read and approved the final manuscript.

\section{Author details}

'Département de Mathématiques, Université de Sherbrooke, Sherbrooke, Québec J1K 2R1, Canada. ${ }^{2}$ Mathematics and Computer Science Department, Laurentian University, Sudbury, Ontario P3E 2C6, Canada.

\section{Acknowledgements}

The authors are grateful to the anonymous referees for their careful reading of the paper and for helpful comments. This work was funded by a grant from the Natural Sciences and Engineering Research Council of Canada.

Received: 10 April 2013 Accepted: 29 August 2013 Published: 06 Dec 2013

\section{References}

1. Murray, JD: Mathematical Biology. Springer, Berlin (1993)

2. Smoller, J: Shock Waves and Reaction-Diffusion Equations. Springer, Berlin (1983)

3. Brezis, $H$, Nirenberg, L: Positive solutions of nonlinear elliptic equations involving critical Sobolev exponents. Commun. Pure Appl. Math. 36, 437-477 (1983)

4. Ambrosetti, A, Brezis, H, Cerami, G: Combined effects of concave and convex nonlinearities in some elliptic problems. J. Funct. Anal. 122(2), 519-543 (1994)

5. Bartsch, T, Willem, M: On an elliptic equation with concave and convex nonlinearities. Proc. Am. Math. Soc. 123(11), 3555-3561 (1995)

6. Cheng, $X, M a, S$ : Existence of three nontrivial solutions for elliptic systems with critical exponents and weights. Nonlinear Anal. 69, 3537-3548 (2008)

7. Wu, TF: On semilinear elliptic equations involving concave-convex nonlinearities and sign-changing weight function. J. Math. Anal. Appl. 318(1), 253-270 (2006)

8. Batkam, CJ, Colin, F: The effects of a Concave-Convex nonlinearity in some noncooperative elliptic systems. Ann. Mat. Pura Appl. Published online 21 April 2013 (to appear)

9. Hsu, TS, Lin, HL: Multiple positive solutions for a critical elliptic system with concave-convex nonlinearities. Proc. R. Soc. Edinb., Sect. A, Math. 139(6), 1163-1177 (2009)

10. Shen, Y, Zhang, J: Multiplicity of positive solutions for a semilinear p-Laplacian system with Sobolev critical exponent. Nonlinear Anal. 74(4), 1019-1030 (2011)

11. Han, P: Multiple positive solutions of nonhomogeneous elliptic systems involving critical Sobolev exponents. Nonlinear Anal. 64(4), 869-886 (2006)

12. Wu, TF: The Nehari manifold for a semilinear elliptic system involving sign-changing weight functions. Nonlinear Anal. 68, 1733-1745 (2008)

13. Chen, CY, Wu, TF: The Nehari manifold for indefinite semilinear elliptic systems involving critical exponent. Appl. Math. Comput. 218, 10817-10828 (2012)

14. Hsu, TS: Multiple positive solutions for a critical quasilinear elliptic system with concave-convex nonlinearities. Nonlinear Anal. 71, 2688-2698 (2009)

15. Szulkin, A, Weth, T: The method of Nehari manifold. In: Handbook of Nonconvex Analysis and Applications, pp. 597-632. Int. Press, Somerville (2010)

16. Bouchekif, M, Nasri, Y: On a singular elliptic system at resonance. Ann. Mat. Pura Appl. 189(2), 227-240 (2010)

17. Chabrowski, J: The Neumann problem for semilinear elliptic equations with critical Sobolev exponent. Milan J. Math. 75, 197-224 (2007)

18. Batkam, CJ, Colin, F: Generalized fountain theorem and application to strongly indefinite semilinear problems. J. Math. Anal. Appl. 405, 438-452 (2013)

19. Kryszewski, W, Szulkin, A: Generalized linking theorem with an application to a semilinear Schrödinger equation. Adv. Differ. Equ. 3, 441-472 (1998)

20. Willem, M: Analyse Harmonique Réelle. Hermann, Paris (1995)

21. Willem, M: Minimax Theorems. Birkhauser, Boston (1996) 
10.1186/1687-2770-2013-268

Cite this article as: Batkam and Colin: Multiplicity result for a critical elliptic system with concave-convex nonlinearities. Boundary Value Problems 2013, 2013:268

Submit your manuscript to a SpringerOpen ${ }^{\circ}$ journal and benefit from:

- Convenient online submission

- Rigorous peer review

- Immediate publication on acceptance

- Open access: articles freely available online

- High visibility within the field

- Retaining the copyright to your article

Submit your next manuscript at $\gg$ springeropen.com 